\title{
DETECCIÓN E INTERVENCIÓN EN LOS CASOS DE DISCONFORMIDAD Y CONFLICTOS DE LAS PERSONAS MAYORES USUARIAS DEL SERVICIO DE AYUDA A DOMICILIO (SAD)
}

\section{DETECTION AND INTERVENTION IN CASES OF DISCONFORMITY AND CONFLICT EXPERIENCED BY ELDERLY PEOPLE USING THE IN-HOME CARE SERVICE}

\begin{abstract}
Alfredo Bohórquez Rodríguez (1) $M^{a}$ José Gómez Martín (1) $M^{a}$ Soledad López Fernández (1), Emma Real González (1), Rocío García Jiménez (1), Marta Del Barrio Sánchez (1), María Prudencio Fernández (1), Marta Sánchez Sánchez (1) $M^{a}$ Teresa Herrero Valdunciel (1) Pilar Serrano Garijo (2) Paloma Jiménez Díaz-Hoyuelo (2) y Bárbara García Figueroa (2)

(1) Eulen Servicios Sociosanitarios (2) Ayuntamiento de Madrid
\end{abstract}

Resumen: El Servicio de Ayuda a Domicilio puede ser definido como un programa individualizado, de carácter preventivo y rehabilitador, que incluye atención personal, doméstica, de apoyo psicosocial y relaciones con el entorno. En este artículo se analizan 62 casos de disconformidad y conflictos de las personas usuarias y sus familias con el servicio concedido o recibido de ayuda a domicilio (realizado en 6 distritos del municipio de Madrid y con un total de 11179 personas usuarias); se expone la realización de grupos de mejora con 729 auxiliares de ayuda a domicilio y la efectividad de dichos grupos para conocer e intervenir en los casos de disconformidad y conflictos descubiertos; y por último se explica cómo se abordaron dichos casos hasta su mejoría o resolución completa, llegando a varias conclusiones, entre ellas, que con una detección precoz de los conflictos y un programa específico, la intervención del SAD es más efectiva.

Palabras Clave: Ayuda a domicilio, Conflicto, Auxiliares SAD, Grupos de mejora, Calidad asistencial.

Abstract: The In-Home Care Service (Servicio de Ayuda a Domicilio, SAD) can be defined as a customized preventive and rehabilitative programme that includes assistance with everyday activities, domestic tasks, psychosocial support and improved interactions with the person's surroundings. This article analyses 62 cases of disconformity and conflict registered by elderly persons who use the in-home service or their families (in 6 districts of the municipality of Madrid and with a total of 11179 users). It describes the process used to address the problems (the creation of improvement groups with 729 in-home care workers) and the effectiveness of said groups for understanding and intervening in the cases of disconformity and conflict detected. Finally, it explains how such cases were handled until they showed significant improvement or were completely resolved. Among other conclusions, it is suggested that the early detection of conflicts and a specific programme to address such conflicts can make In-Home Care intervention more effective.

Key Words: In-home care, Domiciliary care, Conflict, In-home care workers, Improvement groups, Care quality.

| Recibido: 12/09/2013 | Revisado: 28/02/2014 | Aceptado: 01/04/2014 | Publicado: 31/05/2014 | Correspondencia: Alfredo Bohórquez Rodríguez. Eulen Servicios Sociosanitarios. Dirección postal: Calle Valle de Tobalina, 56. CP 28021. Madrid. Teléfono: 916310808 / 629075548. E-mail: abohorquez@eulen.com.

Referencia normalizada: Bohórquez, A., Gómez, M.J., López, M.S., Real, E., García, R., Del Barrio, M., Prudencio, M., Sánchez, Herrero, M.T., Serrano, P., Jiménez, P., y García, B. Detección e intervención en los casos de disconformidad y conflictos de las personas mayores usuarias del Servicio de Ayuda a Domicilio (SAD). Trabajo Social Hoy, 72,7-22. doi. 10.12960/TSH.2014.0007. 


\section{INTRODUCCIÓN}

El Servicio de Ayuda a Domicilio (SAD), es un programa individualizado, de carácter preventivo y rehabilitador, que incluye atención personal, doméstica, de apoyo psicosocial y relaciones con el entorno (Rodríguez y Valdivieso, 1997: 34). La finalidad del servicio, es apoyar a las personas para que puedan permanecer en sus propios domicilios con un adecuado nivel de calidad de vida, reforzando sus potencialidades para su autonomía, la convivencia personal y las relaciones sociales (García, 2010: 8).

Según el estudio del Ayuntamiento de Madrid realizado sobre sus datos de actividad de 2005, el perfil tipo de usuario de SAD, es una mujer de 80 años que vive sola, cuya red social se encuentra deteriorada, es frágil o en situación de dependencia (Ayuntamiento de Madrid, 2005: 46).

La calidad del servicio tiene relación con todo el proceso del servicio, desde su diseño y características, la concesión del tipo de prestación que dé respuesta a las necesidades de la persona usuaria, como la información recibida y el tipo de atención realizada por los/las profesionales del servicio. En este sentido, el servicio incluye un proceso de valoración y asignación del tipo de recurso que cada persona usuaria va a recibir (prestaciones, intensidades, tareas), un proceso de alta y asignación del profesional que realizará los apoyos y cuidados (auxiliar SAD), un proceso de atención y un seguimiento para la mejora del servicio y resolución de incidencias. En todos estos procesos la información, el trato y la calidad de la atención son claves en la prestación del servicio.

Según el documento "Propuesta de mejora del diseño del SAD", realizado por un grupo de trabajo interinstitucional y multiprofesional coordinado por ASADE (Asociación Empresarial de Ayuda a Domicilio), se considera imprescindible aportar a las personas usuarias y sus familias una descripción general del servicio, con lo que se incluye y no se incluye, compromisos, derechos y obligaciones (Bohórquez et al., 2010: 3). Esta información, puede facilitar la comprensión del servicio y clarificar lo que la persona tiene derecho a recibir y las condiciones del servicio que se deben respetar.

En este sentido, la Sociedad Española de Geriatría y Gerontología, en su publicación de "100 recomendaciones básicas para la mejora continua del diseño y funcionamiento del servicio de atención a domicilio", de forma adicional a los derechos, incluye una relación de deberes de las personas usuarias, dentro de los que se destacan, cumplir con los aspectos definidos en su plan de atención y no encomendar al/ a la auxiliar cualquier tarea que no figure en el mismo. También resalta el deber que tiene la persona usuaria de comportarse con corrección, respeto y cordialidad en el trato con las personas que atienden el servicio (Bohórquez et al., 2006: 12). 
Uno de los aspectos más determinantes en la calidad de atención percibida por la persona usuaria, son las actuaciones profesionales, el trato y atención realizada por el profesional auxiliar de ayuda a domicilio. El presente estudio quiere cuantificar los casos de conflictos, quejas o demandas que presentan las personas usuarias con respecto a la prestación concedida (desacuerdos al querer tareas o frecuencias diferentes a las que le han concedido) o conflictos con la prestación recibida (por conflictos con la auxiliar de ayuda a domicilio o por algún otro aspecto relacionado con la prestación del servicio). En todo este proceso multifactorial, influyen aspectos propios de la persona usuaria (su estado de salud y necesidades) y aspectos de los/as profesionales (la información que dan, el trato y el tipo de respuesta y seguimiento ante conflictos y quejas).

Algunas ideas para evitar conflictos en el servicio, las aporta la norma de calidad española "UNE 158301 de requisitos para la gestión de ayuda a domicilio", la cual resalta dentro de los derechos de las personas usuarias, el derecho a presentar sugerencias, quejas y reclamaciones, el derecho a que se respete el tiempo establecido y se cumplan las tareas según el protocolo establecido u orden de prestación. Igualmente, recomienda que se disponga de un protocolo de quejas y reclamaciones y un protocolo de medición de satisfacción y mejora (AENOR, 2007: 11-15). Por otra parte, en la publicación de AENOR (Asociación Española de Normalización y Certificación) "Satisfacción del Cliente", se resaltan como principios a tener en cuenta en el tratamiento de quejas los siguientes: claridad, imparcialidad, confidencialidad, accesibilidad, exahustividad, equidad y sensibilidad (AENOR, 2010: 87).

Como recomendaciones para dar a los/las auxiliares para evitar conflictos con las personas usuarias, se resaltan las siguientes: saber realizar peticiones, rechazar las demandas que no sean adecuadas, disculparse y admitir los errores propios, cuidar el aspecto personal, cumplir las normas de comportamiento como auxiliar, cumplir las normas del servicio, ser puntual y mantener un posicionamiento ético y deontológico, entre otros (Rodríguez et al., 2011: 188). Igualmente, es importante en la formación y apoyo que reciben los/las auxiliares de ayuda a domicilio, incluir pautas de realización correcta del trabajo, pautas de comunicación, emociones en las personas mayores, la confianza, escucha activa y el respeto (Rodríguez et al., 2011: 60).

En el seguimiento del servicio, además del seguimiento telefónico por parte del equipo de coordinación de trabajadores/ras sociales, son importantes las visitas domiciliarias a las personas usuarias para verificar el cumplimiento de las prestaciones y los objetivos encomendados a las auxiliares, comprobar la adaptación entre la auxiliar y la persona usuaria, dar respuesta a las demandas y necesidades de la persona usuaria y resolver las incidencias (Guillén, 2008: 163). 


\section{METODOLOGÍA DE LA INVESTIGACIÓN}

Previo al estudio y con una duración de un año (junio de 2010 a junio de 2011), se realizaron grupos de mejora de auxiliares del SAD, en los que se planteó como el problema más relevante de la calidad del servicio los casos de disconformidad o conflictos de las personas usuarias con el servicio concedido o recibido. Los grupos de mejora de auxiliares del SAD son un sistema participativo con reuniones entre coordinadores/ras y grupos de auxiliares del SAD, donde los/las auxiliares aportan por escrito cuáles son los problemas más importantes de la calidad del servicio, sus ideas y propuestas de mejora. Se han realizado a lo largo de un año 30 reuniones, con la participación de unas 15-25 auxiliares por grupo, en los que cada persona aportaba individualmente y por escrito sus ideas y opinión, que luego se leían y se ponían en común. Con esta información, posteriormente, el equipo de coordinación del servicio (trabajadores/ras sociales), categorizaban la información y priorizaban las acciones a realizar.

En total participaron y aportaron su conocimiento 729 auxiliares (231 en la detección y priorización de problemas, 267 en la categorización de causas del problema seleccionado y 231 en la propuesta de acciones de mejora). Con la información aportada por los/las auxiliares sobre las causas del problema seleccionado (los conflictos o disconformidades), el equipo de coordinación del servicio categorizó las causas de la siguiente forma:

- La persona usuaria tiene desconocimiento por falta de información, no le queda claro o debido a su estado de salud, no comprende las características del servicio que tiene concedido. Implica a los canales de información de los servicios sociales municipales y de la entidad prestadora del servicio. Representan el 64,92 \% de respuestas de los/las auxiliares.

- La astucia de la persona usuaria hace que, aun conociendo las condiciones del servicio que tiene asignado, busque la forma de saltarse las normas establecidas en el servicio para conseguir una prestación diferente o superior a la que tiene concedida. Representan el 25,23 \% de respuestas de los/las auxiliares.

- Existencia de un trato peyorativo hacia la auxiliar del SAD, por considerarla personal de servicio doméstico y no una profesional. Esta situación conlleva un abuso en las demandas y exigencias por parte de la persona usuaria. Representan el 8,31\% de respuestas de los/las auxiliares.

Finalmente, los/las auxiliares y el equipo de coordinación de los grupos de mejora, proponen como acciones de mejora las siguientes:

- Con los responsables y titulares del servicio, revisar la documentación a entregar a las personas usuarias, convivientes y familiares, para que contenga información 
detallada del servicio que evite los conflictos (incluyendo, tareas, derechos y deberes de las personas usuarias, tareas excluidas del servicio, frecuencia de tareas, etc.).

- Realizar visitas domiciliarias frecuentes por parte de los/las coordinadores/ras, incidiendo en la explicación de funciones de la auxiliar y clarificando dudas.

- Reforzar el mensaje para que cada auxiliar del SAD cumpla con sus obligaciones, realizando el trabajo según las indicaciones de coordinación.

- Fomentar la implicación de los familiares en estos casos, especialmente si la persona usuaria presenta un deterioro cognitivo, problemas de salud mental o situación de dependencia.

- Realizar un estudio de investigación para compartir el conocimiento de esta experiencia en una publicación.

Posterior a esta fase de grupos de mejora, como fuente de conocimiento, información e ideas, se realizó el estudio en el SAD de Madrid (de titularidad pública del Ayuntamiento de Madrid y gestión privada de la empresa EULEN Servicios Sociosanitarios), en los distritos de Hortaleza, Latina, Chamartín, Chamberí, Centro y Moncloa. Estos distritos tienen 958641 habitantes, un 29,8\% del total de los 3,2 millones de habitantes de Madrid, según datos del Ayuntamiento de Madrid en 2012. El SAD en estos distritos atiende a 11179 personas usuarias. El estudio tiene varias fases que se explican a continuación.

\subsection{FASE DE DISEÑO}

Con una duración de 6 meses y realizada en el segundo semestre de 2011, se realiza el diseño del estudio, de la intervención y el soporte documental. Basándonos en las ideas y propuestas de las auxiliares del SAD, se realizaron varias reuniones entre los equipos de los servicios sociales municipales y los responsables de la coordinación y dirección técnica de la entidad prestadora del servicio. A nivel de soporte documental de registro e intervención, se elaboraron los siguientes documentos:

- Registro de datos: Se cumplimenta previo a la fase de intervención, y al finalizar la misma, la siguiente información: Ciudad. Distrito. Edad. Género. Tiempo de alta en el servicio (en meses). Intensidad horaria mensual actual (horas/mes). Modificación de intensidad en los últimos 6 meses. Número de conflictos en los últimos 6 meses. Cambio de auxiliar en los últimos 6 meses. Estado cognitivo de la persona usuaria (normal o alteración cognitiva o de salud mental). Tiene información escrita y actualizada de prestaciones. Tiene visita inicial con explicación de prestaciones. Tiene visita de seguimiento en los últimos 6 meses. Tiene llamada de seguimiento en los últimos 6 meses. Queja verbal o escrita de persona usuaria/familiar en los últimos 6 meses y número. De quién procede la demanda (usuario/a, familiar, 
conviviente, vecino, otro). Causa de la demanda (detallarla). Causas del conflicto: nueva tarea que no tiene concedida (de atención personal, doméstica o de otra prestación), frecuencia superior a lo concedido, falta de información, no recibir prestación concedida, conflicto con el/la auxiliar, otra causa. Resultado de la intervención: éxito (resolución completa o gran mejoría) y fracaso (resolución moderada-discreta, sin resolución o empeoramiento).

Documento elaborado y que se entrega a las personas usuarias del servicio de ayuda a domicilio (para facilitar la comprensión del servicio y evitar conflictos y malos entendidos). Este documento se entrega a todas las personas usuarias con conflictos. Se acuerda entre la entidad titular y la entidad prestadora del servicio la siguiente información a entregar:

- Con respecto a el/la auxiliar del servicio: el/la auxiliar de ayuda a domicilio realizará las tareas ordenadas por el ayuntamiento y no realizará otras, salvo que lo indique la trabajadora social municipal. El/la auxiliar de ayuda a domicilio no podrá permanecer solo/a en el domicilio de la persona usuaria, ni tener la llave del domicilio. Las compras que realiza el/la auxiliar siempre serán a cuenta de la persona usuaria. La persona usuaria debe facilitar el ejercicio de las tareas del/la auxiliar de ayuda a domicilio que presta el servicio, así como poner a su disposición los medios materiales adecuados para el desarrollo de las mismas. Las actividades encomendadas a el/la auxiliar, no deben suponer un riesgo físico ni peligro para su salud.

- Con respecto al servicio: La prestación del servicio no excederá del tiempo estipulado. Los desplazamientos necesarios para la realización de las gestiones de la persona usuaria, se harán en transporte público (autobuses o taxis), pagando la persona usuaria los gastos correspondientes (de ella misma y de el/la auxiliar). La persona usuaria debe informar al ayuntamiento o al coordinador/a de la empresa, de cualquier cambio en su situación personal, familiar, social y económica, que afecte a la prestación del servicio. La persona usuaria debe comunicar, con suficiente antelación, cualquier ausencia del domicilio que impida la prestación del servicio. Los horarios de prestación del servicio se acordarán entre la persona usuaria y el equipo de coordinación de la empresa, ajustándose a las siguientes franjas horarias determinadas por el ayuntamiento: aseos y levantar antes de las 10 horas. Aseos y acostar de 20 a 22 horas. Preparación de comida de 13 a 15 horas. El resto de prestaciones se adaptarán al horario general del servicio de 7 a 22 horas.

- Servicios excluidos del SAD: No se incluyen en el servicio las prestaciones que requieren especialización, como curas de enfermería, inyectables, colocar o quitar sondas, manipulación de aparatos o instrumentos que precisen de conocimientos sanitarios, suministrar medicación que requiera técnicas especializadas, actuaciones podológicas cuando exista riesgo de salud o diabetes. No incluye la presta- 
ción a otros miembros de la familia o allegados que habiten en el mismo domicilio, la realización de arreglos o mantenimiento de la casa, como empapelado, pintura, etc., y la atención a los animales de compañía.

\subsection{FASE DE INTERVENCIÓN}

Con una duración de 6 meses, se realizó en el primer semestre de 2012. En esta fase se identifican los casos de demandas o quejas de personas usuarias o familiares que hayan tenido conflictos importantes con las tareas y prestaciones concedidas o recibidas de ayuda a domicilio (en más de una ocasión en los últimos 6 meses), y se cumplimenta el registro de recogida de datos, por cada caso, al inicio y al final del período de intervención.

La intervención es coordinada entre los servicios sociales municipales y la entidad prestadora del servicio y se basa en dar información del servicio, realizar visitas de seguimiento a la persona usuaria y dar pautas y apoyo al auxiliar del SAD. En la fase de intervención, la relación de los casos iniciales son reportados a las juntas municipales. Ante casos nuevos, se reporta la información directamente al trabajador social de la junta municipal correspondiente. Las principales áreas y acciones de intervención son las siguientes:

- Desde la entidad titular del servicio: Seguimiento telefónico o presencial por los servicios sociales municipales. Si procede, actuación específica de servicios sociales municipales ante dudas, cambio de intensidad o modificación de la prestación. Confirmar entrega de documentación y su comprensión (carta de servicios del SAD, órdenes de prestación y nuevo documento de aclaraciones).

- $\quad$ Desde la entidad prestadora del servicio: Se realiza una visita de seguimiento del coordinador/a de la entidad prestadora a la persona usuaria (antes de un mes desde la detección del caso), para aclarar la situación y tomar las medidas oportunas (informando de la situación al trabajador/a social municipal). Se explican los aspectos de comunicación y trato con el/la auxiliar, explicación de derechos y deberes, explicar las prestaciones del servicio (incluidos y excluidos), reforzar la implicación de la familia y realizar nueva entrega de documentación del servicio. Nuevos contactos telefónicos, con llamadas del coordinador/a, de explicación y seguimiento. Desde la coordinación de la entidad prestadora, se establecen pautas específicas y de apoyo para los/las auxiliares (reunión de información, formación, reporte de casos, pautas al auxiliar, guía práctica, cumplimiento de prestaciones concedidas, tratamiento de casos muy conflictivos, técnicas de resolución de conflictos, etc.). Si procede, propuesta de modificación del servicio o cambio de auxiliar. 
- Conjunta (entidad titular y prestadora): En las visitas de coordinación y seguimiento periódicas entre trabajador/a social municipal y coordinador/a de la entidad prestadora, se incluye la revisión de los casos de disconformidad. En los casos que se consideren necesarios, se puede valorar una visita conjunta del trabajador/a social municipal y la coordinadora de la entidad prestadora.

\section{RESULTADOS}

De un total de 11179 personas usuarias del servicio de ayuda a domicilio, se detectan 62 casos de disconformidad o conflicto de las personas usuarias con la prestación concedida o recibida (tasa del 0,55\%). Los distritos analizados tienen un rango entre 0,29 \% y 0,92 \%, siendo sus datos los siguientes: Hortaleza (19 casos, 0,92 \%), Latina (20 casos, 0,59\%), Chamartín (5 casos, 0,53\%), Chamberí (4 casos, 0,29\%), Centro (8 casos, $0,41 \%$ ) y Moncloa (6 casos, 0,42\%).

De los 62 casos, se excluyen del análisis final tras la intervención, 4 casos por fallecimiento y 3 por tener menos de 65 años. De las 55 personas mayores usuarias del SAD, con conflictos, se reportan los siguientes datos:

- Datos del grupo de personas usuarias con conflictos o disconformidades: Edad media de 82,24 años, el 90,9 \% ( $n=50)$ mujeres, el 74,5 \% $(n=41)$ tienen estado cognitivo normal y el 70,9\% ( $n=39$ ) habían presentado quejas verbales o escritas. En total, en 6 meses, presentaron 179 conflictos (3,25 por persona) y 112 quejas verbales o escritas (2,03 quejas por persona).

- Casi las $3 / 4$ partes de los casos habían presentado quejas previamente, las propias quejas pueden ser un buen elemento de detección y teniendo en cuenta que, igualmente, las personas de este grupo mayoritariamente tienen buena situación cognitiva, puede ser útil aprovechar que las personas tienen capacidad de comunicación y comprensión para recibir la información, por lo que el asesoramiento, las visitas para aclarar las dudas, comprender las inquietudes que nos manifieste directamente la persona usuaria, nos pueden permitir abordar y solucionar la situación.

- Con respecto a la prestación del servicio, las personas usuarias con conflictos o disconformidades tienen una media de 69 meses de alta en el servicio, una intensidad media mensual de SAD de 28,06 horas, el 92,7 \% ( $n=51$ ) no habían tenido modificación de la intensidad horaria en los últimos 6 meses. El 72,7 \% ( $n=40)$ con información escrita y actualizada, el 96,4 \% ( $n=53$ ) con visita inicial con explicación de prestaciones, el 45,5\% (n=25) con visita de seguimiento en los últimos 6 meses, el 89,1 \% ( $n=49)$ con llamadas telefónicas de seguimiento en los últimos meses (con una media de 2,4 llamadas en 6 meses).

- Dado que la incidencia es baja y el grupo de media lleva más de 5 años en el servicio, parece importante contar con un programa o protocolo específico para reforzar la detección de este tipo de casos e iniciar la intervención lo antes posible. 
- Las principales causas de los conflictos son: En el 72,7 \% de las personas ( $n=40)$ conflictos con la auxiliar, en el 30,9\% $(n=17)$ por tarea no concedida, en el $20 \%(n=11)$ por descontento con el horario, en el 18,2\% (n=10) se detecta trato inadecuado por parte de la persona usuaria o familiar a la auxiliar del SAD, en el $14,5 \%(n=8)$ por cambio de auxiliar, en el 10,9 \% (n=6) por falta de información y en el 3,6 \% (n=2) por no recibir la prestación concedida (ver Gráfico 1, "Tipos de conflictos o disconformidades más frecuentes").

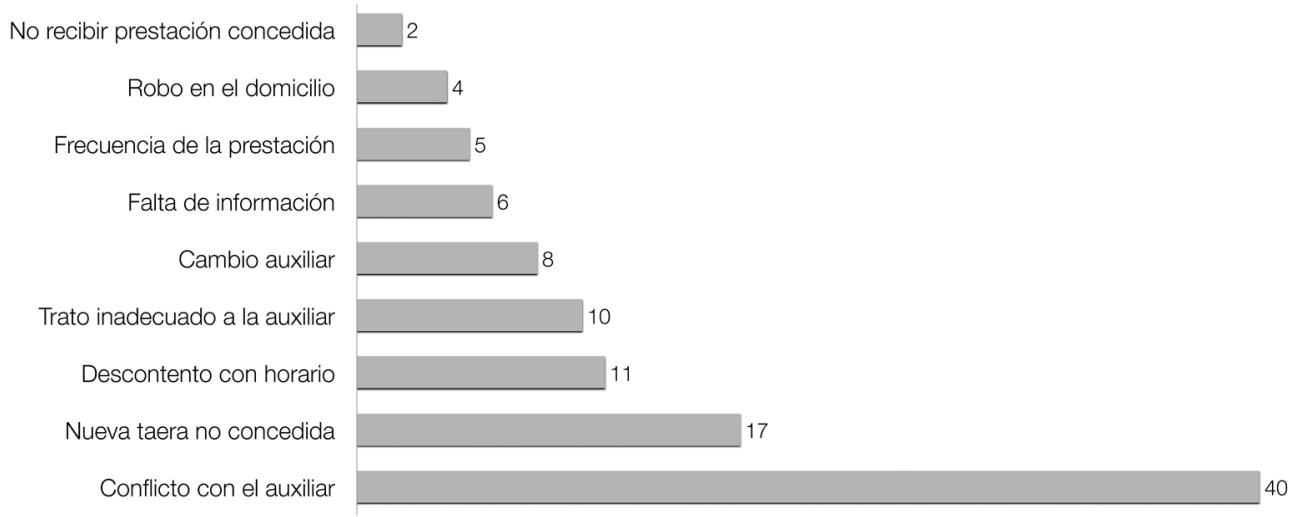

Gráfico 1. Tipos de conflictos o disconformidades más frecuentes.

- En el 60 \% ( $n=33)$ de las personas, los conflictos son reportados por la propia persona usuaria, en el 16,4 \% proceden del familiar $(n=9)$ y en el 23,6 \% proceden de otras personas ( $n=13)$, (ver Gráfico 2, "Persona o entidad que reporta el conflicto").

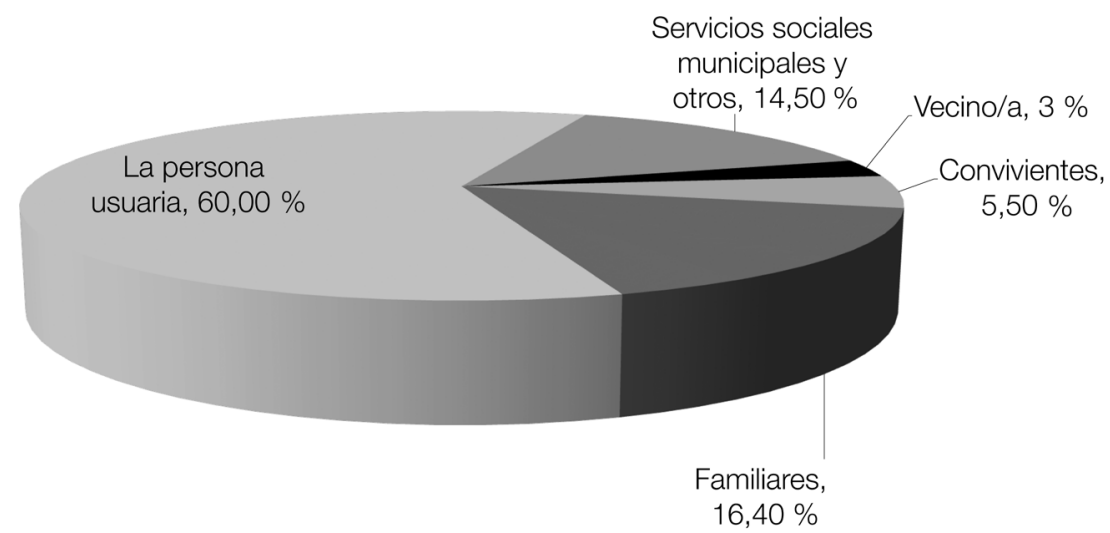

Gráfico 2. Persona o entidad que reporta el conflicto. 
- Un área clave a tener en cuenta en la intervención, es la conformada por todos los aspectos de relación e interacción entre la persona usuaria y la auxiliar del SAD, tanto en temas de información, como en temas de relación, trato o negociación de condiciones, que pueden generar situaciones de malos entendidos, conflictos o disconformidad. En su conjunto, este área debe ser objeto de una doble actuación, tanto con la persona usuaria, como con la auxiliar del SAD.

- Tras la intervención (ver Gráfico 3, "Resultados tras la intervención”), se obtuvo buen resultado (resolución completa o importante mejoría) en el 72,7 \% ( $n=40)$ de los casos y mal resultado (mejoría discreta, sin mejoría o empeoramiento) en el $27,3 \%$ de los casos ( $n=15)$. Estos datos nos indican, que los casos de conflictos o disconformidades de personas usuarias con el servicio concedido o recibido, son un área clave de intervención, de baja prevalencia, que genera altas cargas de trabajo (más de 3 conflictos en 6 meses) y donde se pueden obtener buenos resultados.

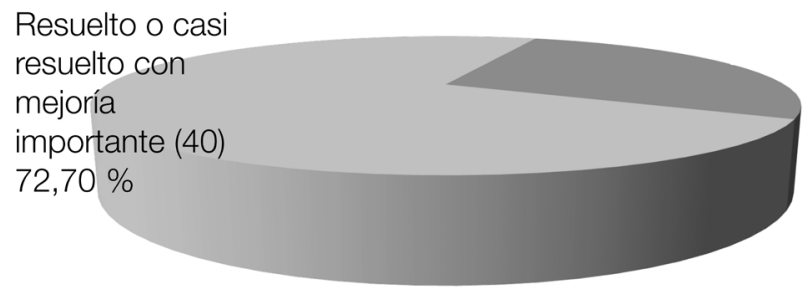

\section{Mejoría}

discreta, no

resuelto o

empeoramiento

(15), $27,30 \%$

Gráfico 3. Resultados tras la intervención.

- Tras la intervención, se entregó información actualizada al 100 \% (n=55) de los casos, se realizó seguimiento telefónico al 100 \%. Por parte de la entidad prestadora del servicio se realizaron nuevas visitas domiciliarias a 43 personas usuarias (78,2 \%) y en 41 casos $(74,5 \%)$, se realizaron visitas específicas por los/las trabajadores/ras sociales de los servicios sociales municipales. Solo en un caso (al no ser necesario), la persona usuaria no fue visitada en su domicilio. Resaltamos la importancia de la información verbal y escrita que se aporta tanto a la persona usuaria como a la auxiliar del SAD y la necesidad de realizar en la fase de intervención, visitas de seguimiento por parte de los/las trabajadores/ras sociales.

- Como otros datos a resaltar, no se realizó ningún cambio en el tipo de prestación, ni ninguna baja por parte de los servicios sociales municipales y el $5,4 \%$ $(n=3)$, presentaron baja voluntaria del servicio. Nos puede indicar que el problema de conflictos no se centra en el tipo de prestación concedida, sino en la información aportada y la prestación recibida. 
- $\quad$ En el 21,8 \% ( $n=12)$, se realizó intervención específica con la familia y en el $27,2 \%(n=15)$, se explicó detalladamente la necesidad del trato hacia el/la auxiliar. En el $40 \%$ de los casos de conflictos con la auxiliar, se realizó tras la intervención un cambio de auxiliar (en 16 de 40 personas). Con respecto al grupo en los que se cambió a la auxiliar ( $n=16)$, el $56,2 \%$ de los casos $(n=9)$, presentó una gran mejoría o resolución tras el cambio. Si bien, previo al inicio, el 52,7 \% ( $n=29$ ) había tenido cambio de auxiliar en los 6 meses previos (con una media de 2,2 cambios por persona usuaria), durante la fase de intervención se cambió la auxiliar al $29 \%$ de los casos $(n=16)$. En estos casos, con una media de más de 5 años de duración del servicio, el cambio de auxiliar es una consecuencia de la situación de conflicto entre la persona usuaria y auxiliar (puede ser solicitado por la persona usuaria, por la propia auxiliar o indicado desde la coordinación del servicio como una acción de tratamiento al problema como recurso final en los casos de no poder resolverse con información, apoyo a la persona usuaria y apoyo a la auxiliar).

- El análisis estadístico de los resultados del estudio, se realizó tras finalizar la fase de intervención en el segundo semestre de 2012 y su finalidad era analizar los diferentes parámetros estudiados entre los dos grupos de resultados de la intervención, el de conflictos resueltos o con gran mejoría (éxito) y el constituido por los casos en los que no se había conseguido un resultado satisfactorio (fracaso).

Los datos se ofrecen de modo descriptivo como frecuencias y medidas de centralización dependiendo del tipo de variable. Para el análisis, en comparaciones de otras variables cualitativas [estado cognitivo (alterado o no) o cambio en la auxiliar (si/no), modificación de intensidad (si/no), visita de seguimiento o tipo de conflicto (con la auxiliar u otro)] con la dicotómica éxito/fracaso, se han hecho tablas de contingencia 2×2, utilizando Chi-Cuadrado para conocer su significación estadística.

En el caso de variables cuantitativas (edad, número de conflictos, tiempo de alta en el servicio o intensidad horaria) se ha hecho la comparación entre medias para muestras independientes (t-Student). Se ha utilizado el paquete estadístico SPSS 17.0. Se obtuvieron los siguientes resultados:

- Se asocian negativamente como factores condicionantes del resultado y con significancia estadística (ver Tabla 1, "Factores condicionantes del resultado"), las personas usuarias con alteraciones cognitivas o problemas de salud mental $(p<0,05)$ y el número previo de conflictos en los últimos 6 meses $(p=0,00)$. En los casos de éxito (resolución o gran mejoría) la media de conflictos en 6 meses es de 2,39 y el $80,5 \%$ de los casos tenían estado cognitivo normal. En los casos de fracaso (mejoría discreta, no resuelto o empeoramiento), la frecuencia es de 6,2 conflictos y el $50 \%$ de los casos tenían alterado el estado cognitivo. En la intervención hay 
que valorar como elementos que influyen en un resultado negativo, el número de conflictos y el estado cognitivo alterado, que dificulta la comunicación y la relación entre persona usuaria y los profesionales del servicio. En estas situaciones puede ayudar a mejorar los resultados, iniciar la intervención lo antes posible y reforzar las acciones de formación y apoyo a las auxiliares que atienden personas con alteraciones cognitivas.

- No se observan diferencias significativas en el resultado, los factores de género, tiempo de alta del servicio, cambio de auxiliar, visitas domiciliarias, edad, ni intensidad horaria.

Tabla 1. Factores condicionantes del resultado

\begin{tabular}{|c|c|c|c|c|c|}
\hline $\begin{array}{l}N^{\circ} \text { CONFLICTOS } \\
\text { EN } 6 \text { MESES }{ }^{*} \\
{ }^{*} P=0.000\end{array}$ & Resolución & $N$ & Media & $\begin{array}{l}\text { Desviación } \\
\text { típ. }\end{array}$ & $\begin{array}{l}\text { Error típ. } \\
\text { de la media }\end{array}$ \\
\hline & Éxito & 40 & 2,39 & 1,178 & 0,196 \\
\hline & Fracaso & 15 & 6,2 & 4,329 & 1,118 \\
\hline \multirow{7}{*}{$\begin{array}{l}\text { ESTADO COGNITIVO* } \\
{ }^{*} P<0.05\end{array}$} & & & Fracaso & Éxito & Total \\
\hline & Normal & $N$ & 8 & 33 & 41 \\
\hline & & $\begin{array}{l}\% \text { dentro } \\
\text { de "Estado } \\
\text { cognitivo" }\end{array}$ & $19,50 \%$ & $80,50 \%$ & $100 \%$ \\
\hline & Alterado & $N$ & 7 & 7 & 14 \\
\hline & & $\begin{array}{l}\% \text { dentro } \\
\text { de "Estado } \\
\text { cognitivo" }\end{array}$ & $50,00 \%$ & $50,00 \%$ & $100 \%$ \\
\hline & Total & $N$ & 15 & 40 & 55 \\
\hline & & $\begin{array}{l}\% \text { dentro } \\
\text { de "Estado } \\
\text { cognitivo" }\end{array}$ & $27,30 \%$ & $72,70 \%$ & $100 \%$ \\
\hline
\end{tabular}

Fuente: Datos del propio estudio.

Hay que tener en cuenta que siendo el grupo general muy amplio (más de 11000 personas usuarias del SAD) y dada la baja prevalencia del problema, el tamaño del grupo de intervención ( $n=55$ ) es pequeño, es posible que ampliando el estudio al $100 \%$ del Área del Ayuntamiento de Madrid, obtengamos un tamaño superior y se puedan observar resultados de nuevos factores condicionantes (positivos y negativos). 


\section{DISCUSIÓN Y CONCLUSIONES}

Los conflictos de las personas usuarias con el servicio concedido o recibido, son un área donde la intervención es efectiva, ya que se obtiene buen resultado (resolución o gran mejoría) en el $72,7 \%$ de los casos. Es recomendable que el programa de intervención incluya dentro de sus ejes y diseño, el apoyo, información y formación al auxiliar, apoyo, información e intervención (visitas al domicilio y apoyo telefónico) con la persona usuaria y su familia, y pautas de comunicación, coordinación y actuación entre la entidad prestadora de servicio y la entidad titular.

Si bien la prevalencia del problema de conflictos o disconformidades de las personas usuarias con respecto al servicio concedido o recibido es baja (el 0,55 \%), es necesario disponer de un sistema de detección de casos y tener definido un plan de intervención conjunto y consensuado entre la entidad prestadora del servicio y la entidad titular del mismo. Por otra parte, a pesar de su prevalencia baja, sus consecuencias generan altas cargas de trabajo (55 casos, generaron 179 conflictos y 112 quejas). Es necesario en todos los casos detectados desplegar un plan de intervención específico y, de esta forma, disminuir el número de conflictos, disconformidades y quejas posteriores.

La mayor parte de los conflictos se da en casos de personas con situación cognitiva y mental normal (en el 74,5\% de los casos), por lo que es necesario reforzar con ellas la comunicación de las características del servicio, los derechos y deberes, al igual que dejar claro de forma verbal y por escrito, los servicios que se excluyen. De esta forma se podrán evitar conflictos innecesarios entre la persona usuaria y el/la auxiliar.

Siendo la media de aparición de los casos de conflictos 69 meses tras el alta $(5,7$ años), es recomendable en los casos de personas usuarias que lleven más de 4 años, hacer un recordatorio de las condiciones y volver a entregar la información actualizada del servicio.

La causa más frecuente son los conflictos con las auxiliares (en el 72,7 \% de los casos) y teniendo en cuenta que el equipo profesional de auxiliares del SAD, es quien mejor conoce la situación real de la prestación del servicio, es muy importante el apoyo que podamos darles, así como promover su implicación tanto en el diseño (grupos de mejora para detección de problemas, análisis de causas y propuestas de acciones de mejora), como en la intervención (pautas, información, formación y seguimiento).

La segunda causa en orden de frecuencia (en el 30,9\% de los casos), son los conflictos por tareas no concedidas. Teniendo en cuenta que en ningún caso fue necesario modificar la prestación, este tipo de conflictos se resuelve con la visita de seguimiento, llamadas telefónicas y con la información aportada de condiciones del servicio, derechos, deberes y tareas excluidas. 
A pesar de no ser una de las causas más frecuentes, en el 18,2 \% de los casos se detectó un trato inadecuado de la persona usuaria o familiar hacia la auxiliar del SAD. En estos casos, es muy importante resaltar como básico y condición ética de partida, el máximo respeto entre las personas. Es necesario apoyar al auxiliar y desde el equipo de coordinación, abordar este tema en el propio domicilio, aclarando su importancia y aportando criterios que faciliten su mediación y resolución (el/la auxiliar debe sentirse apoyado/a).

Una de las opciones de intervención ante los conflictos con el/la auxiliar y ante la aparición de estrés profesional, puede ser realizar el cambio de auxiliar (fue necesario en la fase de intervención en casi un $30 \%$ de los casos). Tras realizarlo, en el $56 \%$ de estos casos se presentó una gran mejoría o resolución del conflicto. De los datos del estudio se observa que los cambios de auxiliar son una consecuencia del problema (en el $52 \%$ de los casos) y no una causa del mismo (lo es solo en el $14 \%$ de los casos).

En el estudio, se han detectado como factores condicionantes negativos en el resultado, los siguientes:

- La alteración cognitiva o problemas de salud mental. Es importante tener en cuenta que en estos casos, es clave la formación especializada de los/las auxiliares del SAD, una adecuada asignación de los/las auxiliares (especialmente en el nivel de conocimientos y compatibilidad con la persona usuaria) y contar con el apoyo continuo desde los/las trabajadores/ras sociales, para evitar el agotamiento y estrés profesional del equipo de auxiliares. En estos casos se hace imprescindible la implicación de la familia.

- El segundo factor es el número de conflictos previo, por lo que es necesario contar con una detección precoz del problema y un protocolo de actuación que incluya todas las pautas, criterios e información a entregar y explicar. Igualmente, debemos iniciar lo antes posible la intervención con un seguimiento telefónico y visita en el domicilio. Es clave mantener una reunión de coordinación y apoyo con el/la auxiliar, para analizar la actuación.

Finalmente, en el estudio se destaca que no hay diferencias significativas en el resultado, por razón de edad, género, tiempo de alta en el servicio, cambio de auxiliar, visitas domiciliarias, ni intensidad horaria. Si bien no encontramos significancia estadística dentro de los factores de condicionamiento positivo en el resultado (posiblemente por el tamaño de la muestra), es necesario resaltar, como los casos con las tasas más elevadas de resolución o gran mejoría, la falta de información (en el 83 \% de los casos), nueva tarea no concedida de otra prestación (en el 75 \%) y nueva tarea no concedida de tipo doméstico (en el $63 \%$ ).

Los conflictos o disconformidades de las personas usuarias con el servicio concedido o recibido tienen una baja prevalencia, generan altas cargas de trabajo y representan un área donde con una detección precoz y un programa específico, la intervención es muy efectiva. 


\section{BIBLIOGRAFÍA}

AENOR. (2007). Norma UNE 158301: Requisitos de gestión del servicio de ayuda a domicilio. Madrid: Ediciones AENOR.

AENOR. (2010). Satisfacción del cliente. Madrid: Ediciones AENOR.

Ayuntamiento de Madrid. (2005). Jornadas 2005: "Servicios de Ayuda a Domicilio, la dependencia en casa". Madrid: Área de Gobierno y Servicios a la ciudadanía. Ayuntamiento de Madrid.

Bohórquez, A., et al. (2006). 100 recomendaciones básicas para la mejora continua del diseño y funcionamiento del servicio de atención a domicilio. Madrid: Grupo de trabajo de calidad. Sociedad Española de Geriatría y Gerontología.

Bohórquez, A., et al. (2010). Propuesta de mejora del diseño del servicio de apoyo a domicilio. Madrid: ASADE.

García, G. (2010). El servicio de ayuda a domicilio en la encrucijada: análisis y reflexiones sobre el presente y futuro del servicio de ayuda a domicilio, tras la implantación del Sistema de Atención a la Dependencia. Madrid: Asociación Estatal de Directoras y Gerentes de Servicios Sociales.

Guillén F., (2008). Síndromes y cuidados en el paciente geriátrico. Madrid: Editorial Elsevier Masson.

Rodríguez, P., y Valdivieso, C. (1997). El servicio de ayuda a domicilio: Manual de formación para auxiliares. Madrid: Sociedad Española de Geriatría y Gerontología y Fundación Caja de Madrid. Editorial Panamericana.

Rodríguez, P., Bermejo, L., Marín, J.M., y Valdivieso C., (2011). Servicio de Ayuda a Domicilio: manual de planificación y formación. Madrid: Serie Gerontología Social. Sociedad Española de Geriatría y Gerontología. Editorial Panamericana. 
